\title{
Characterization of Nancy Grace Roman Space Telescope slitless spectrometer (grism)
}

\author{
Qian Gong $\odot,{ }^{\mathrm{a}, *}$ Matthew Bergkoetter $\odot,{ }^{\text {a }}$ Joshua Berrier, ${ }^{\text {a }}$ \\ Victor J. Chambers, ${ }^{a}$ Margaret Dominguez, ${ }^{\text {a }}$ Wesley Fincher, \\ John Hagopian, ${ }^{\text {c }}$ Catherine Marx, ${ }^{\text {a }}$ and Laurie Seide ${ }^{d}$ \\ ${ }^{a}$ NASA Goddard Space Flight Center, Greenbelt, Maryland, United States \\ ${ }^{\mathrm{b}}$ Tech Innovations LLC, Glenwood, Maryland, United States \\ ${ }^{c}$ Lambda Consulting, LLC, Harwood, Maryland, United States \\ ${ }^{\mathrm{d}} \mathrm{KBR}$, Greenbelt, Maryland, United States
}

\begin{abstract}
We have published the optical design and early test results of the Roman Space Telescope grism spectrometer in previous SPIE proceedings. We report the follow-on activity of the spectral and radiometric calibrations, including the calibration methods, experiment designs, and test equipment calibration, such as the light source and detectors used in the test. The grism calibration includes the throughput versus wavelength, which is largely determined by the diffraction efficiency of the two diffractive surfaces. It also includes spectral resolution, point spread function, and relative radiometric measurements. The measured results are presented. The comparisons between the test data and the theoretical simulations are also presented. The tests and results presented are from the engineering test unit in ambient room temperature environment. The thermal/vacuum tests are planned to verify the results when the flight unit is ready. (C) The Authors. Published by SPIE under a Creative Commons Attribution 4.0 Unported License. Distribution or reproduction of this work in whole or in part requires full attribution of the original publication, including its DOI. [DOI: 10.1117/1.JATIS.6.4.045008]
\end{abstract}

Keywords: grism; slitless spectrometer; grating; diffraction efficiency; spectral resolving power; encircled energy.

Paper 20040 received Apr. 28, 2020; accepted for publication Dec. 9, 2020; published online Dec. 26, 2020.

\section{Introduction}

The Nancy Grace Roman Space Telescope (RST) is a NASA observatory designed to unravel the secrets of dark energy and dark matter, search for and image exoplanets, and explore many topics in infrared astrophysics. Roman has a 2.4-m telescope, the same size as the Hubble Space Telescope (HST), but with a field of view (FOV) 100 times greater than HST. ${ }^{1}$ The Roman Space Telescope will serve as an important tool for the science community through its General Observer programs. All Roman data will be publicly available immediately after processing and delivery to the archive. By submitting proposals through the competitive program, scientists around the world will also be able to use the observatory to study the cosmos in their own way, from the nearest exoplanets out to clusters of distant galaxies.

Roman includes two main instruments: the Wide Field Instrument (WFI) and the CoronaGraph Instrument (CGI). The WFI includes high-resolution and low-resolution slitless spectrographs, and a number of carefully selected bandpass filters. The high-resolution spectrograph is a grism assembly. ${ }^{2}$ It consists of two diffractive surfaces and two powered prisms. The low-resolution spectrograph is a prism assembly, which consists of two powered prisms. ${ }^{3}$ The emphasis of this paper is to discuss the spectral and radiometric calibration and characterization of the grism spectrograph.

The paper is arranged as follows. Section 2 briefly describes the Engineering Test Unit (ETU) grism design. It also describes the calibration and characterization set up, the equipment used in the set up, and the radiometric and wavelength calibration accuracy. It also discusses the optical

*Address all correspondence to Qian Gong, qian.gong-1@nasa.gov 
design of the testbed and the baseline wavefront quality of the testbed. Section 3 is dedicated to grism diffraction efficiency and throughput measurements. Section 4 focuses on wavelength dispersion scale measurements and provides the dispersion scale fit. Section 5 is dedicated to spectral resolving power and encircled energy as a function of field position, wavelength, and detector pixel size. Section 6 examines the relative radiometric measurements of the signal from the wanted order of the grism diffractive surfaces and the background noise from the unwanted orders of the diffracted surfaces. In Sec. 7, the test results are summarized and compared to the Roman grism requirements.

\section{Calibration and Characterization Setup}

The ETU grism design used the same principle as the prototype described in Ref. 1. The assembly is in noncollimated space. A second diffractive surface compensates the geometric aberrations created by the first diffraction surface. The schematic of the ETU optics is shown in Fig. 1. The detailed information on ETU alignment and test is described in Hagopian et al. ${ }^{4}$

Grism spectral calibration is very critical and challenging because a slitless spectrometer does not have a slit as a wavelength reference. For the slitless spectrograph, the spectra can be everywhere in the entire FOV depending upon the object location. For some slitless spectrographs with low spectral resolution, the grism only needs a small wedge angle to provide the required spectral resolution. In that case, the zeroth order from the grating does not have obvious dispersion introduced by the prism wedge. The zeroth-order image can then be used as a reference, as is done for the HST grism. However, the spectral resolution of the Roman grism is much higher and the element is designed to be zero deviation for the central wavelength. The dispersion from the two prisms broadens the point spread function (PSF) of the zeroth order in the dispersion direction to $\sim 38$ pixels for the full wavelength range. Therefore, the only references in the image are the edges of the wavelength band for the Roman grism. To measure an object's red shift, which is one of the science goals of Roman, the required total spectrum wavelength accuracy is $1 \mathrm{~nm}$; only $0.1 \mathrm{~nm}$ is allocated to grism calibration. Unlike some very highresolution spectrographs used for radial velocity (RV) measurements, where $0.1 \mathrm{~nm}$ corresponds to a number of pixels on the detector array, the $0.1 \mathrm{~nm}$ for the Roman grism corresponds to only $1 / 10$ of a pixel. Examples of RV spectrographs include the Echelle spectrograph NEID on the WYIN telescope of Kitt Peak Observatory, ${ }^{5}$ and the Echelle spectrograph on the Vainu Bappu Telescope. $^{6}$

Based on the requirement, we selected a super-continuum source (NKT's SuperK) as the light source for the entire calibration. The SuperK has a "monochrometer" called SELECT. The SELECT covers the wavelength range from 640 to $>2000 \mathrm{~nm}$, a great fit to the grism and

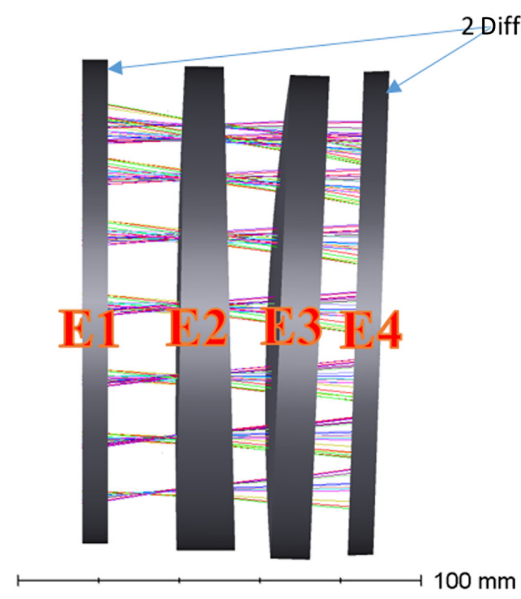

(a)

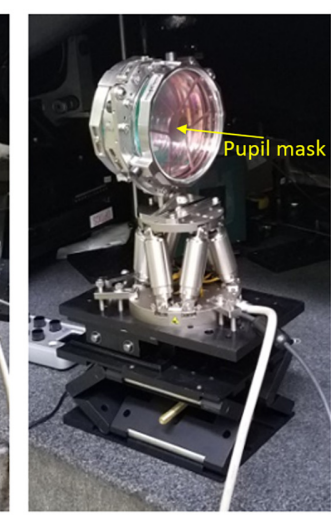

(b)

Fig. 1 (a) Grism schematic. E1 and E4 are optical flats with a diffractive surface on each of their back surfaces. E2 and E3 are powered prisms. (b) Assembled ETU grism. 
prism wavelength range. The grism ranges from 1000 to $1930 \mathrm{~nm}$, and the prism ranges from 750 to $1800 \mathrm{~nm}$. The SuperK SELECT can have up to eight output wavelength lines simultaneously within the wavelength range. The line width is a weak function of the wavelength. On average, the full-width at half-maximum (FWHM) is around $10 \mathrm{~nm}$. The SuperK CONNECT couples the SELECT output into an endlessly photonic crystal single-mode fiber that is used as a diffraction limited point source for the testbed.

The SuperK and its SELECT component are used for diffraction efficiency, throughput, and relative radiometric measurements because the 10-nm FWHM is acceptable. However, the 10$\mathrm{nm}$ FWHM is too wide for encircled energy and spectral resolving power measurements. The dispersion of the grism spreads the 10-nm band to $\sim 9$ pixels, significantly affecting encircled energy and spectral resolving power measurements. Fiber tunable narrowband filters and comb filters with an FWHM $<0.5 \mathrm{~nm}$ provide less dispersed PSFs.

An optical simulator mimics the beam of the Roman telescope that enters the grism. Our simulator testbed is a single elliptical mirror. Its layout is shown in Fig. 2. The point source is at one of the elliptical foci (the focus that is closer to the ellipse) and the detector is at the other focus. The ellipse is designed in such a way that the $f / \#$ of the ellipse output beam is at $f / 7.2$, which provides a larger cone angle than the Roman telescope's $f / 7.8$ beam. Therefore, the pupil mask inside the grism assembly is fully illuminated. The projection of the Roman central obscuration and struts are also built into the pupil mask.

The $f / \#$ of the ellipse is smaller than that of the telescope, a fundamental requirement that guarantees that the ellipse simulator will satisfy the Roman telescope/grism interface. The simulator is fixed to the optical table for alignment stability, limiting the test configuration to a single-field angle. Therefore, the grism must be positioned correctly relative to the ellipse to simulate different field angles. This is achieved by positioning the grism relative to the ellipse such that the chief ray from the ellipse intersects the first surface of E1 at the same angle and relative position as the chief ray from the telescope for each selected field. The advantage of this approach is that the distortion introduced by the Roman telescope is simulated by the chief ray angle and position.

WaveFront Error (WFE) is another factor used to qualify the ellipse as a valid telescope simulator. The as-designed off-axis ellipse is aberration free. Due to mirror fabrication and alignment error, the measured WFE is $41 \mathrm{~nm}$ rms on average, which is a little below the residual WFE of Roman telescope over the field average. (The RST telescope residual has an obvious field dependence due to its large FOV. Since the magnitude of the WFE is well below the diffraction limited WFE for the entire FOV and in full wavelength range, the simulator for the spectral and radiometric calibration does not simulate the telescope residual wavelength.) The emphasis of this paper is to concentrate on the grism calibration and characterization. The testbed design, metrology, and verification will be detailed in another paper.

The elliptical mirror and the point source (the tip of the fiber) are carefully aligned to optimize the wavefront. The low-loss, single-mode fibers large mode area (LMA)-5 and LMA-8 from NKT are selected to deliver the light from SELECT to the shorter focus of the ellipse. LMA fibers are photonic crystal fiber and are endlessly single-mode fiber. The LMA-5 fiber has a large NA $=0.2$ at $\lambda=1064 \mathrm{~nm}$. The $f / \#$ of the fiber is a function of wavelength. The NA equals 0.36 at the longest wavelength $1930 \mathrm{~nm}$, which still overfills the ellipse. Nyquist sampling of the PSF is important. The LMA-8 fiber with a smaller NA $=0.14$ was also used to make the

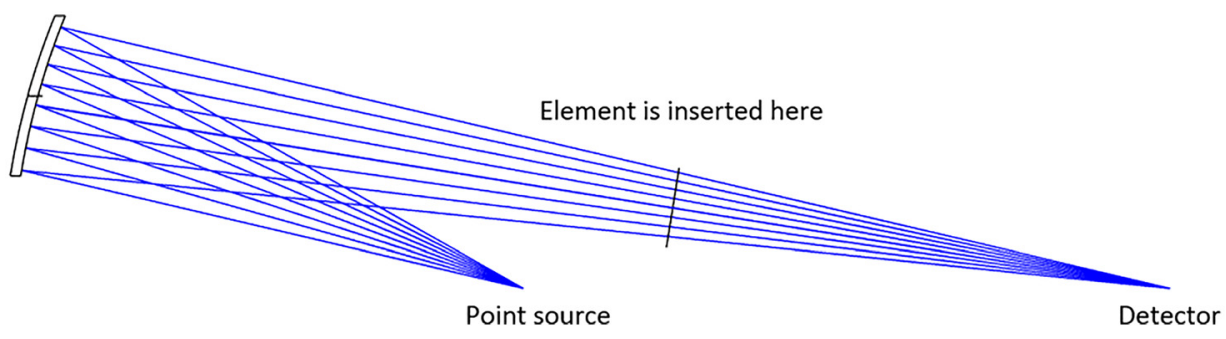

Fig. 2 The elliptical mirror test bed. It provides a $\sim f / 7.2$ beam, which fully covers the grism aperture $(f / 7.8)$. 


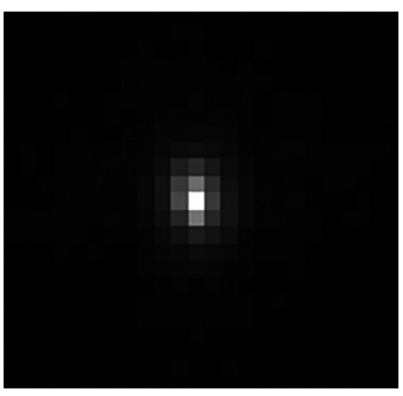

Fig. 3 PSF at $1543 \mathrm{~nm}$. The Airy disk diameter for this $f / 7.2$ system should be $\sim 27 \mu \mathrm{m}$. The detector pixel is $12.5 \mu \mathrm{m}$. This shows diffraction limited performance.

beam more Gaussian apodized. This helps to increase the PSF for a better Nyquist fit at the shorter wavelength range. Figure 3 shows the measured PSF from the testbed itself. The diffraction limited performance of the testbed has been demonstrated. During testing, the ellipse is fixed. The grism is tilted to simulate the angle of the focal plane relative to grism in Roman telescope/grism design.

The set up in Fig. 2 is the baseline configuration for the grism and prism calibration and characterization. The only differences for each test are the source spectral profiles, the detectors, the detector position, and the grism position and orientation.

Due to the spectral response of our IR detector array, a Sensors Unlimited InGaAs detector array with $12.5-\mu \mathrm{m}$ pixel pitch, the tests currently only cover from 1000 to $1650 \mathrm{~nm}$. A detector array that covers the full wavelength range from 1000 to $2600 \mathrm{~nm}$ has been ordered.

\section{Diffraction Efficiency and Throughput Measurement}

The requirement for the throughput efficiency is the following.

The grism assembly mean throughput efficiency shall be greater than or equal to 0.55 over the operational angle of incidence range, within the FWHM passband when in the optical path with mean efficiency ( 0.55$)$ over wide bandpass ( $98 \%$ of passband) and minimum efficiency (0.35) over the bandpass.

For the grism, the throughput efficiency is dominated by the diffraction efficiency. As mentioned in Gong et al., ${ }^{2}$ two diffractive surfaces are used to compensate the grating introduced wavelength-scaled aberration. The grism diffraction efficiency is the multiplication of the two diffraction grating efficiencies. Figure 4 shows a theoretical diffraction efficiency curve as a

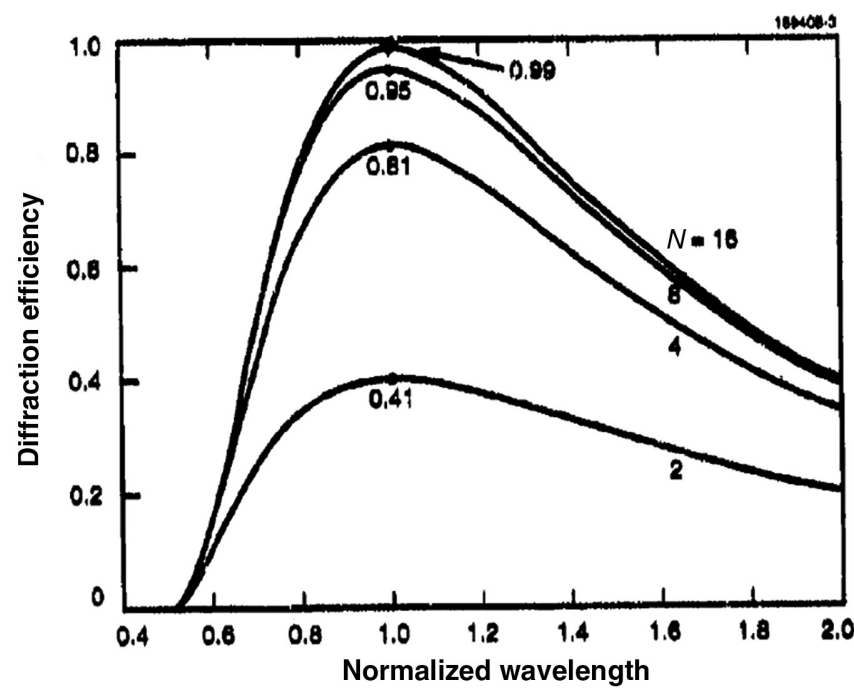

Fig. 4 The first-order diffraction efficiency of a phase grating as a function of wavelength and for varying phase levels. ${ }^{8}$ The numbers 2,4 , and 8 are $N=2, N=4$, and $N=8$. 
function of wavelength for a single multilevel diffractive surface. ${ }^{8}$ It is seen that $N=16$ provides the highest diffraction efficiency. Conveniently, $N=16$ is commonly used in the dry etch fabrication processes on fused silica substrates and was used in this grism. Considering the full grism wavelength range (1000 to $1930 \mathrm{~nm}$ ), the peak efficiency wavelength was designed to be at $1330 \mathrm{~nm}$. The diffraction efficiencies at $\lambda_{\min }=1000 \mathrm{~nm}$ and $\lambda_{\max }=1930 \mathrm{~nm}$ are the same at $70 \%$. After including the two diffractive surfaces, the theoretical maximum at $\lambda_{\min }$ and $\lambda_{\max }$ is $49 \%$, not including the material absorption loss, antireflection (AR) coating loss, or bandpass filter loss.

The grism diffraction efficiency measurement set up is shown in Fig. 5. Note: it is called diffraction efficiency measurement for Engineering Development Unit (EDU) and throughput for Environmental Test Unit (ETU). The difference between the EDU and ETU assemblies is only that the ETU assembly has a bandpass coating on the second grism element (E2). The source for this measurement is the SuperK plus the SELECT. The narrowband tunable filter was not used for this test, because the diffraction efficiency of the grism is a slowly varying function of wavelength. The wavelength range for this test was 1000 to $1900 \mathrm{~nm}$. The monochromatic light out of the SELECT was coupled into a single-mode fiber with the fiber tip carefully positioned at the near focus of elliptical mirror. The diffraction efficiency of the EDU includes the material absorption and AR coatings on all surfaces. After the EDU test, the second element in the EDU was replaced by an identical one but with a bandpass coating on one surface and an out-of-band blocking coating on the other side. Because the EDU and ETU are the same except for the bandpass filter, the set up is the same for both. The output power for the source fluctuates, especially when the wavelength is changed. A reference detector is used to normalize the signal in a method similar to what is done in commercial spectrometers. In this set up, two identical photodiodes, which cover the wavelength range of 900 to $2600 \mathrm{~nm}$, are used.

The diffraction efficiencies for both ETU and EDU were measured at three selected field positions: central field, $X$ field $=-10 \mathrm{deg}$, and $Y$ field $=-3 \mathrm{deg}$. Note that the field listed here is the chief ray angle that enters the grism not the field from the sky. The spectral dispersion is in the $y$-direction (vertical direction). The test results for the three selected fields are plotted in Figs. 6(a), 6(b), and 6(c).

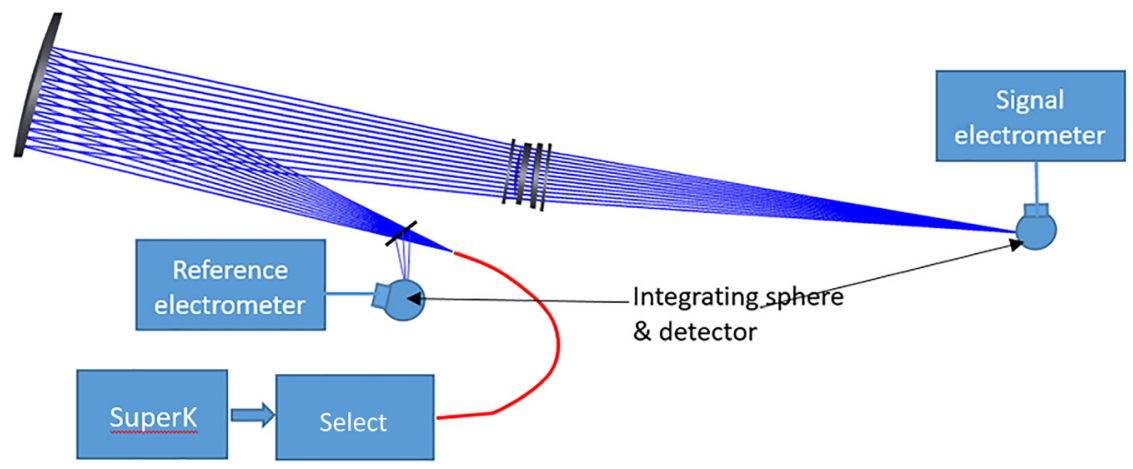

(a)

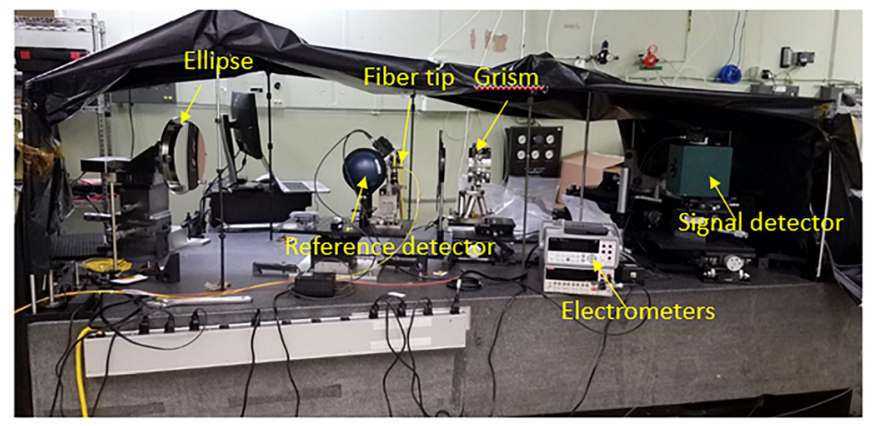

(b)

Fig. 5 (a) Grism diffraction and throughput measurement set up and (b) a picture of the set up. 


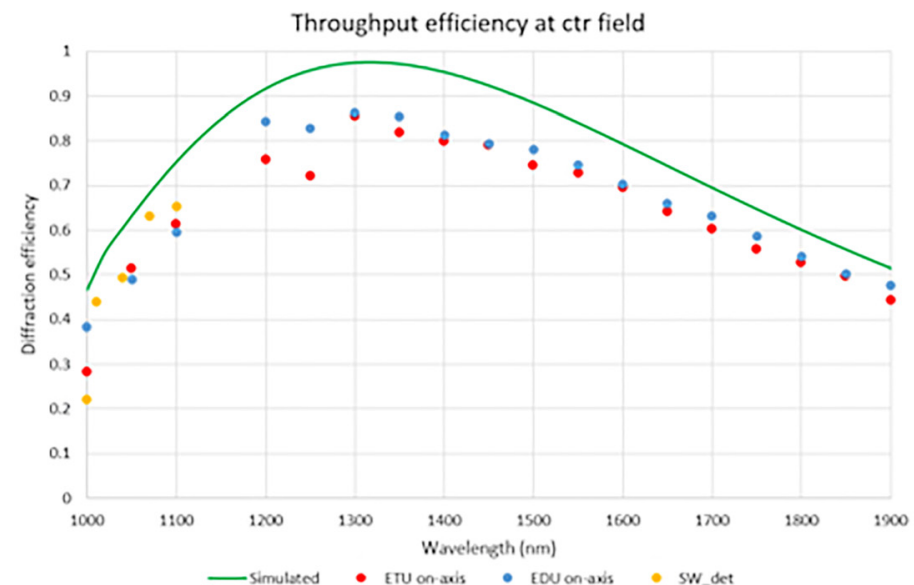

(a)

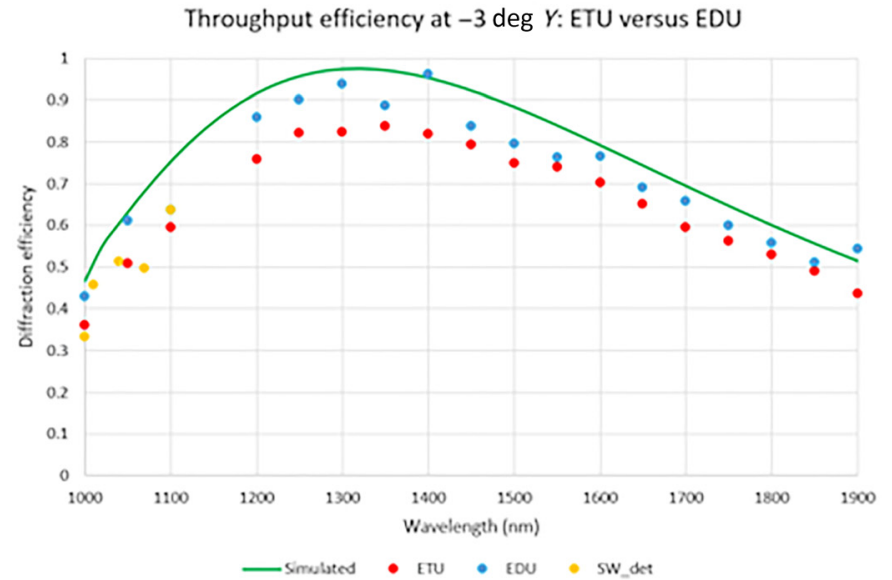

(b)

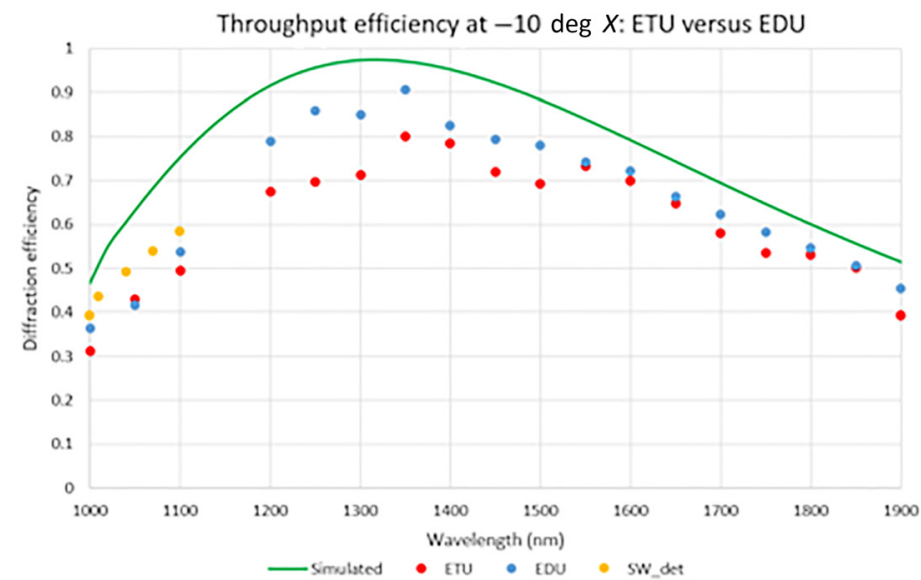

(c)

Fig. 6 (a) The throughput efficiency at the central field. The green line on the plot is the simulated curve that only includes the diffraction efficiency of the two diffractive surfaces using the asdesigned model. (b) The throughput efficiency at 3 deg in the $-Y$ direction (dispersion direction). (c) The throughput efficiency at 10 deg in the $-x$ direction (nondispersion direction).

The blue dots are the measured efficiency of the EDU that includes the diffraction efficiency, material absorption, and AR coating loss. The red dots are the measured efficiency of the ETU. As mentioned above, the difference between ETU and EDU is just a bandpass coating on the second element of the ETU. The yellow dots are the same measurement as the red dots, but using two 

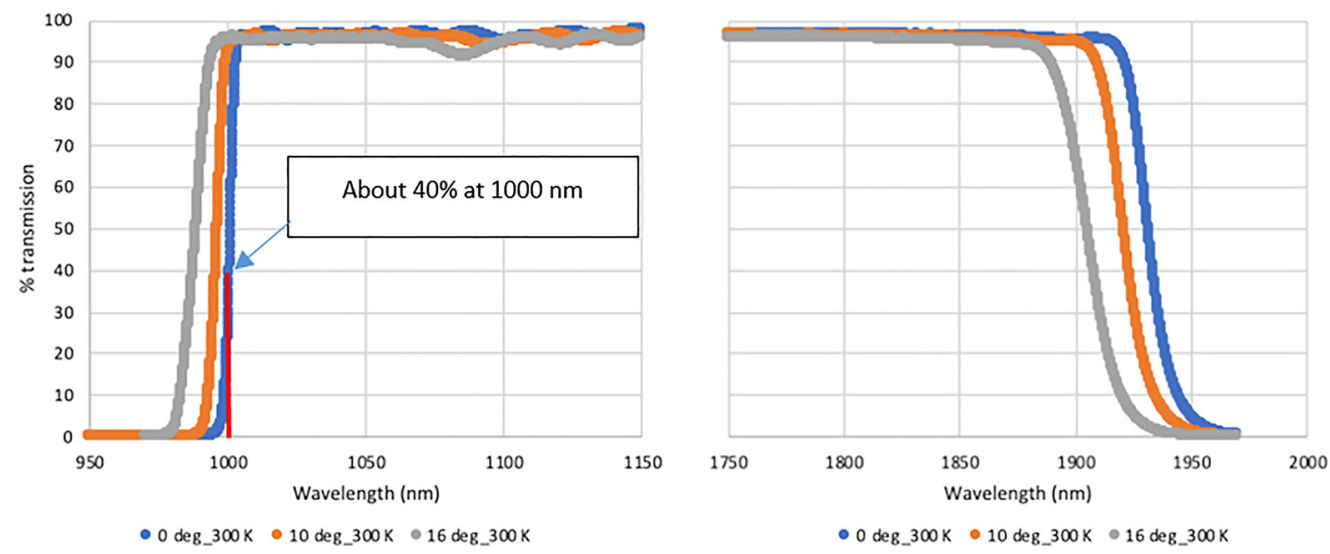

Fig. 7 Measured ETU bandpass coating sample: transmittance versus wavelength.

different InGaAs photodiodes optimized over the 800 to $1700 \mathrm{~nm}$ range to improve the signal-tonoise ratio. The step size is smaller to show the blue edge effect of the bandpass coating.

Also of note is the efficiency at the blue edge of the bandpass. The yellow dots with the finer step size show that the filter blue edge is a function of incident angle. The efficiency increase from 1000 to $1010 \mathrm{~nm}$ is the largest for the central field and the smallest for the $-10 \mathrm{deg}$ in $Y$ field. The bandpass edge shifts to a shorter wavelength when the incident angle increases, as expected from thin film coating theory.

\subsection{Comparison to Requirements}

The measured result shows that the required mean efficiency of 0.55 over wide bandpass is met and exceeded. The minimum efficiency of 0.35 for EDU is a little short for the blue end of ETU. This is not due to diffraction efficiency drops, but because the transmittance of the filter edge is at $\lambda=1000 \mathrm{~nm}$ (see Fig. 7). If we use the theoretical diffraction efficiency value 0.47 at $1000 \mathrm{~nm}$, then multiply $40 \%$ transmission at $1000 \mathrm{~nm}$, the measured throughput $\sim 0.2$ is very reasonable. The revised requirement has shifted the starting wavelength to $1010 \mathrm{~nm}$.

\section{Dispersion Magnitude (Scale)}

Dispersion scale is critical to the grism calibration. Because of the nature of the slitless spectrometer, on-orbit calibration is only able to determine the blue and red edges of the spectrum. The rest of the wavelength range relies on the interpolation from the ground calibrated dispersion scale. The dispersion scale is a function of wavelength and field position. The geometric distortion and dispersion nonlinearity are combined. Spectral traces have different dispersion lengths and tilt orientations as a function of field. In some field positions, the spectral traces are slightly curved. Figure 8 shows the simulated spectral dispersion from 1000 to $1930 \mathrm{~nm}$ for different field positions.

Spots are shown at $1,1.25,1.55,1.75$, and $1.93 \mu \mathrm{m}$. It is noted that the maximum and minimum dispersion lengths at different field positions differ by $\sim 10 \%$. The orientation differences and "smile" shape can also be seen.

To measure the dispersion scale, an IR detector array is needed to image the PSF at sample wavelengths. This measurement was limited by our current detector array, which has good spectral response only from 1000 to $1600 \mathrm{~nm}$.

The three spectral traces highlighted in green frames in Fig. 8 are the three selected fields. These three fields were selected because the wavefront had previously been measured at these same fields. They are not really the most interesting because the dispersion in the three fields does not change much in magnitude. A similar method will be used to map the dispersion for additional field angles at a later date.

The measurement set up is similar for the throughput efficiency measurement. No reference detector is needed and the photodiode at the focal plane is replaced by a Sensors Unlimited IR 
Gong et al.: Characterization of Nancy Grace Roman Space Telescope slitless spectrometer (grism)

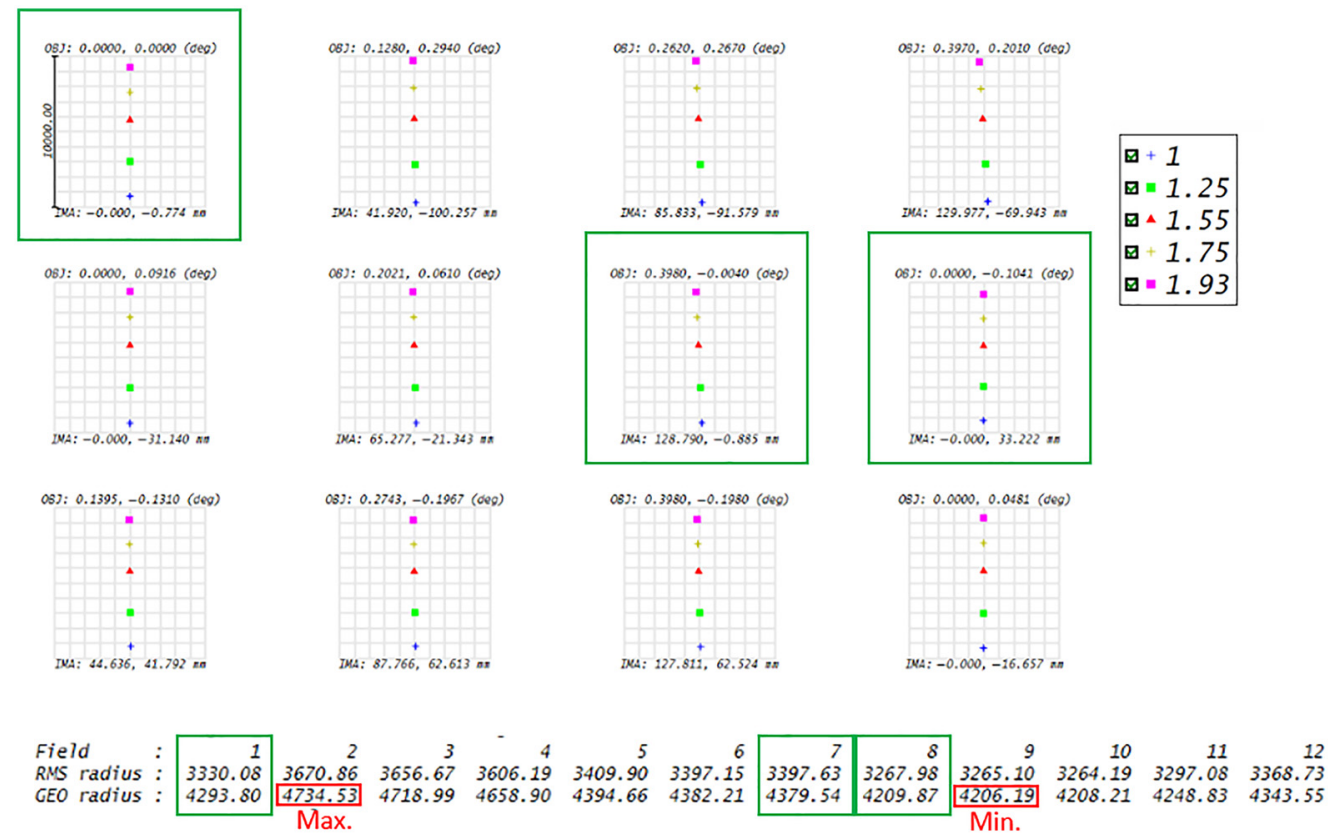

Fig. 8 Baseline spectral dispersion from EDU optical design for 12 different field positions.

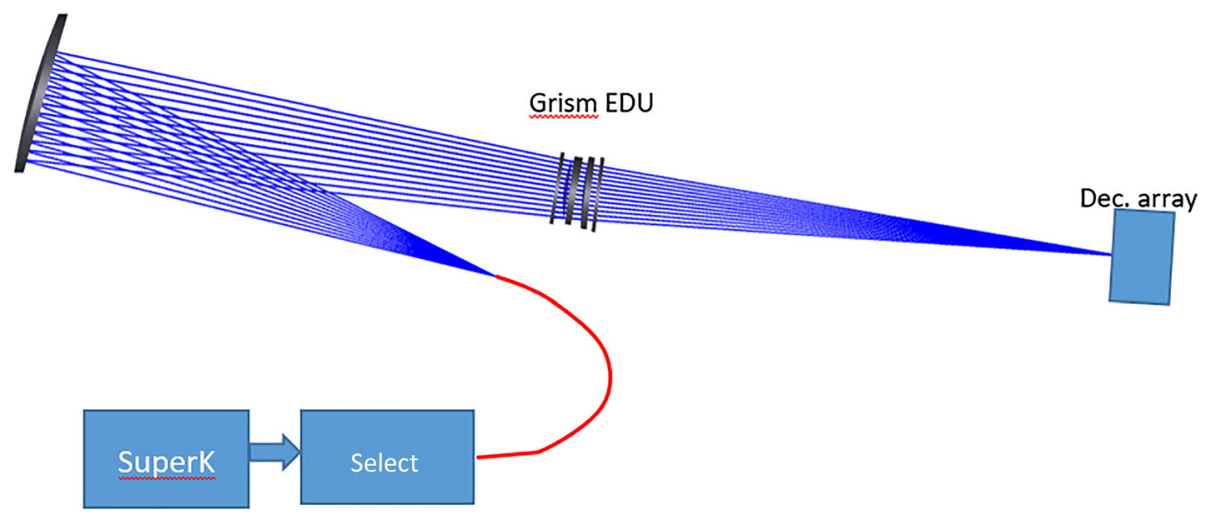

(a)

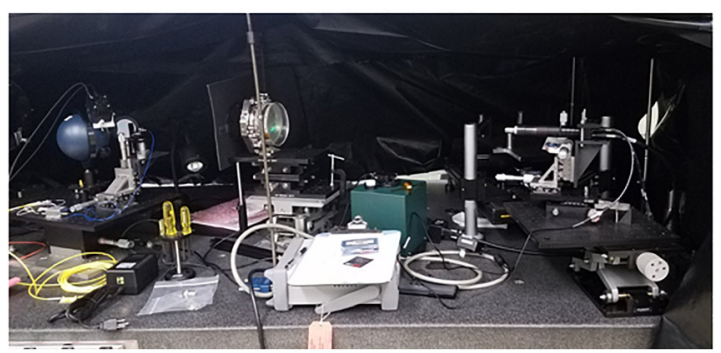

(b)

Fig. 9 (a) The dispersion scale measurement set up and (b) the photo of the set up. The grism tips and tilts to simulate the desired field position.

detector array (Fig. 9). The IR detector has a pixel size of $12.5 \mu \mathrm{m}$, a little larger than the $10-\mu \mathrm{m}$ Roman pixels.

Since the SELECT uses two fiber connectors to deliver the full wavelength range, the images were taken in two wavelength ranges (1000 to $1100 \mathrm{~nm}$ and 1200 to $1600 \mathrm{~nm}$ ). The images are shown in Fig. 10. 


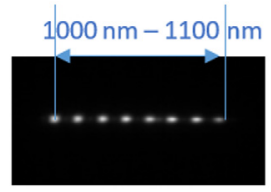

(a)

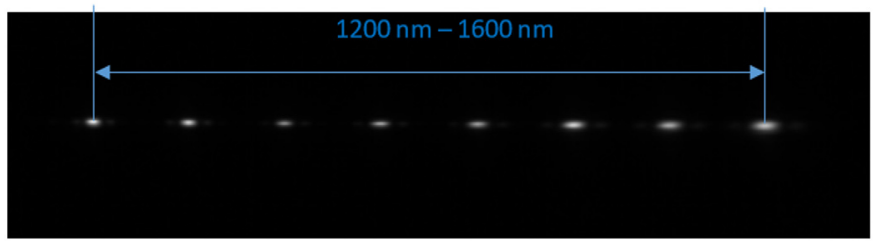

(b)

Fig. 10 (a), (b) Spectral dispersed point source on an IR detector array. It is noted that the spot size is elongated in the horizontal direction. This is caused by the FWHM of each spectral line from the SELECT. Note that the bandwidth of each line from the SELECT varies and becomes wider for longer wavelengths.

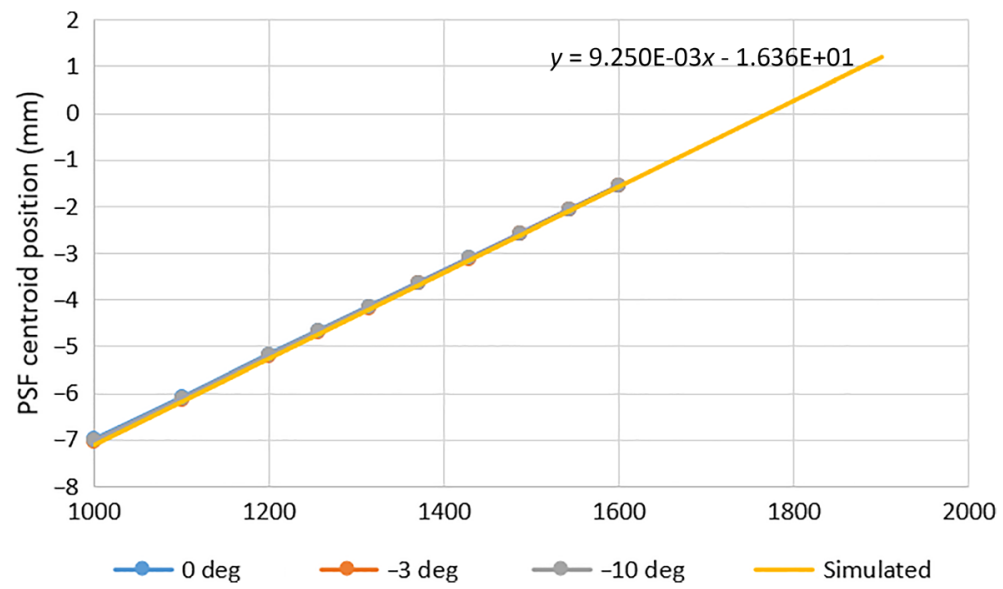

Fig. 11 Modeled and measured dispersion scale. The modeled curve is for the central field at 0-deg, 0-deg, and 3-deg lines are overlapped with the simulated line.

During data analysis, the two images were stitched together based on metrology data to provide the full dispersion scale measurement. The dispersed PSF centroid position versus wavelength, the dispersion scale, is plotted in Fig. 11. The equations of the linear fit of Fig. 11 are listed in Table 1.

The requirement of the grism spectral dispersion scale is shown below.

The grism spectral dispersion shall be between 1.0 and $1.2 \mathrm{~nm} /$ pix over all wavelengths and the FOV.

Table 1 Dispersion scale at different field positions.

\begin{tabular}{lcc}
\hline \hline & Dispersion scale $(\mathrm{nm} / 10-\mu \mathrm{m}$ pix $)$ & Dispersion equation $(\mathrm{mm} / \mathrm{nm})$ \\
\hline FOV: (0 deg, 0 deg) model & 1.081 & $9.250 \times 10^{-3} x-1.636 \times 10^{1}$ \\
FOV: (0 deg, 0 deg) measured & 1.105 & $9.047 \times 10^{3} x-1.603 \times 10^{1}$ \\
FOV: (-10 deg, 0 deg) measured & 1.103 & $9.068 \times 10^{-3} x-1.606 \times 10^{1}$ \\
FOV: (0 deg, $-3 \mathrm{deg})$ measured & 1.092 & $9.157 \times 10^{-3} x-1.621 \times 10^{1}$ \\
\hline \hline
\end{tabular}


The dispersion scale in Table 1 shows that the spectral dispersion requirement is met for all measured fields. However, the current accuracy does not meet the science goal for analyzing spectral lines from the grism at $0.1 \mathrm{~nm}$ accuracy in the newly added requirement. To achieve the 0.1-nm spectral accuracy from the slitless spectrometer (grism), the following tests are planned: (1) accurate bandpass filter transmission measurements will be made at a number of selected field positions. (2) Accurate dispersion scale versus wavelength measurements will be made at the same selected fields. A polynomial fit from these two measurements will provide the blue and red edges, as well as the dispersion scale to cover the full FOV. Given the field angle of the object, the wavelength of all lines can be obtained from the polynomial.

\section{Spectral Resolving Power and Encircled Energy}

Note: The test and results presented in this section are to provide scientists a proof-of-concept verification with the available hardware in hand. Further tests are planned with new hardware aimed to achieve confirmation of the newly added requirement.

The spectral resolution measurement set up is shown in Fig. 12. It is nearly identical to the set up for dispersion scale measurements except a narrow band fiber tunable filter is inserted between the SELECT and the fiber. The narrow band tunable filter is crucial for measuring the spectral resolution and the encircled energy. The grism has the dispersion scale of $\sim 1 \mathrm{~nm} / \mathrm{pix}$. The spectral line from SELECT has the FWHM $\sim 6$ to $10 \mathrm{~nm}$ depending upon the wavelength. This means that the PSF will be 6 to 10 pixels long in the dispersion direction. Such an elongated PSF in the dispersion direction makes a Nyquist resolving power measurement impossible. A narrow band tunable filter cuts the 10-nm FWHM spectral lines to $\sim 1 \mathrm{~nm}$ FWHM, enabling the resolving power and encircled energy measurement.

The interval between two adjacent lines is designed as $3 \mathrm{~nm}$, which includes two pixels for Nyquist plus one pixel for the line width from the source. This is due to the source having a 1-nm FWHM and the larger pixel size of the detector (12.5 $\mu \mathrm{m}$ compared with the flight detector pixel size of $10 \mu \mathrm{m})$. Three tunable fiber narrowband filters are currently available with the central wavelengths at 1040, 1310, and $1550 \mathrm{~nm}$. Each covers an $80 \mathrm{~nm}$ bandwidth. The spectral resolving power is measured in each band. Figure 13 shows the spectrum taken using the

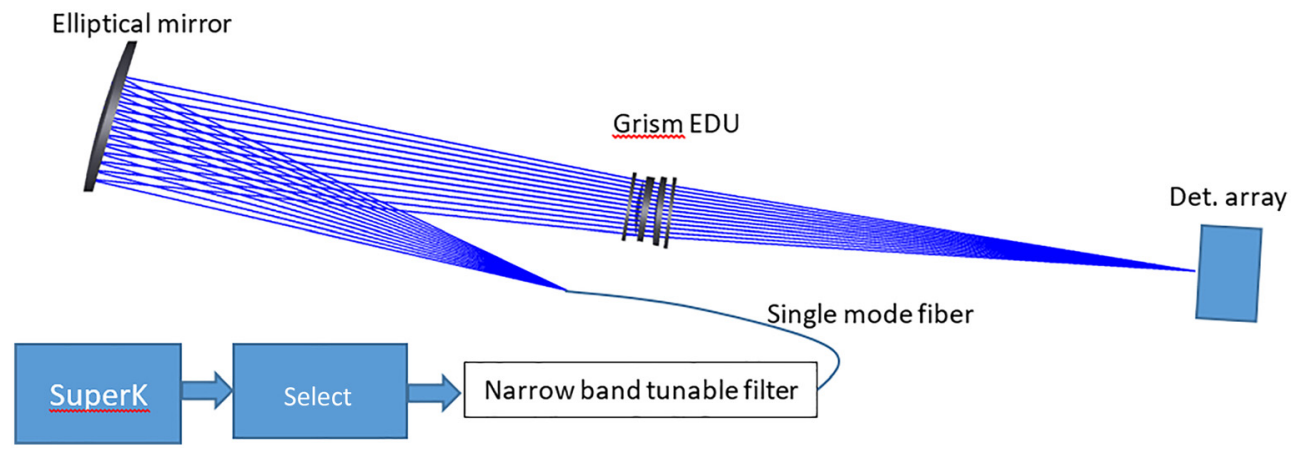

Fig. 12 Spectral resolving power and encircled energy measurement setup.

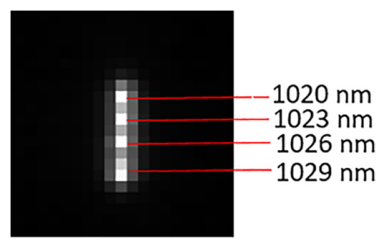

Fig. 13 The spectrum of EDU grism at $\lambda \approx 1025 \mathrm{~nm}$. 
IR camera for wavelength lines 1020, 1023, 1026, and $1029 \mathrm{~nm}$. Each wavelength was carefully adjusted using a ThorLabs optical spectrum analyzer. The spectrum indicates that the Nyquist condition is met even with $12.5-\mu \mathrm{m}$ pixels.

The data around 1310 and $1550 \mathrm{~nm}$ were also taken, but the spectrum was not resolved. This is not caused by the grism, but due to the following reasons: (1) The main error is the tilt angle of the image plane relative to grism axis. Even though the source is a point source, the full spectrum is $\sim 8 \mathrm{~mm}$ long, so the tilt angle must be considered. (2) The detector pixel size of $12.5 \mu \mathrm{m}$ is larger than the flight detector size of $10 \mu \mathrm{m}$. (3) The Airy disk size is proportional to the wavelength. Based on the first-order principles, the PSF size is larger than Nyquist sampled PSF size at the longer wavelengths.

Once we test the grism again, we plan the following changes. For item 1, a tilt bracket will be used to ensure the spot is always in focus no matter which wavelength or field position is measured. For item 2, we plan to use an IR microscope objective to magnify the PSF to reduce the pixelation effect. For item 3, we are going to perform analysis and create a lookup table of minimum resolved $\Delta \lambda$ versus wavelength and verify it in the lab.

The encircled energy has not been tested yet. This measurement will also require a microscope objective to magnify the image on the IR camera. We are looking forward to completing these tests after the ETU completes its cryowavefront test.

\section{Relative Radiometric Calibration}

This section describes the relative radiometric calibration of the grism. As mentioned previously, the grism assembly has two diffractive elements. Therefore, it has many more orders than the grism on HST. It has the wanted order $(1,1)$, and unwanted orders $(0,0),(1,0),(-1,0),(1,2)$, $(-1,2) \ldots$; the square of what the HST has. The main purpose of this section is to assess the background for all unwanted orders. Fortunately, our grating efficiency is very high and the PSF size of most unwanted orders is more than a few orders of magnitude larger than the PSF of the wanted order. The unwanted orders can be divided into two categories: (1) the same diffraction orders from both diffractive surfaces: $(0,0),(-1,-1),(2,2),(-2,-2)$, etc. and (2) cross orders, meaning that the PSF is generated from different orders of the two diffractive surfaces: $(1,-1)$, $(1,0),(1,2),(0,1),(2,1)$, etc.

For category \#1, the PSF of these orders are relatively small compared to the cross orders for category \#2, but are still much larger than that of the wanted order. Since they are from unwanted orders from both diffractive surfaces, the diffraction efficiency is much lower, making them less of a concern. The dispersion due to the prisms in the grism assembly disperse the $(0,0)$ order PSF to a 38-pixel-long image for the full wavelength range, reducing the impact from the standpoint of ghost analysis. The downside, however, is that this zero order cannot be used for guiding.

For category \#2, only the order combinations with a wanted order ( +1 order) from one of the two diffractive surfaces are considered. The other combinations can be ignored because they carry little power and are under the detector noise level. These cross-order PSFs are much larger than the diffraction orders discussed above.

\subsection{Category \#1 PSF from Unwanted Diffraction Orders}

The purpose of this test is to determine the relative intensities of a wanted PSF and category \#1 PSF. The lab set up schematic is shown in Fig. 12 and is the same as the set up for the resolving power measurement except the source is monochromatic.

The designed wavelength range for the grism is from 1000 to $1930 \mathrm{~nm}$. However, the available IR camera used during the measurement has good sensitivity only from 900 to $1600 \mathrm{~nm}$. Therefore, the wavelengths selected for this test are: 1010, 1340, and $1580 \mathrm{~nm}$. Further testing will include the full wavelength range. Among all orders in category \#1, the order $(0,0)$ has the highest peak intensity and smallest PSF size, because all other orders have grating-introduced dispersion. In other words, it is the worst case for ghosting, and the best potential for telescope guiding. We also had limited time to perform this test, therefore, it is the only category \#1 order we compared to the wanted order. 
Due to the limited detector dynamic range and the significant intensity difference between wanted and unwanted orders, also because our detector chip is not big enough to capture both $(0,0)$ and $(1,1)$ orders, the images are stitched together from two frames. First, a PSF image of the $(0,0)$ order is taken with the peak pixel intensity at or over $2 / 3$ full well. The peak intensity is then recorded for the ratio calculation, Finally, the detector is moved such that the $(1,1)$ order PSF is on the same detector position and another image is taken using the same source intensity and same exposure time. The detector nonuniformity error is minimized by having both PSFs at the same position of the detector. The second image is enormously saturated at the peak. The detector position is then kept fixed and the exposure time is gradually reduced while taking a series of images until the peak of the $(0,0)$ order PSF is no longer saturated. The saved images of $(1,1)$ order PSF is analyzed by comparing the counts of the same pixel from two consecutive images. The pixel selection is arbitrary as long as the counts are from two consecutive frames in the linear range of the detector. The ratio of the two counts indicates the intensity reduction

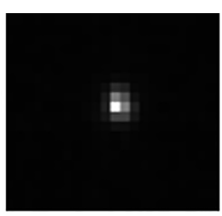

$a-1$

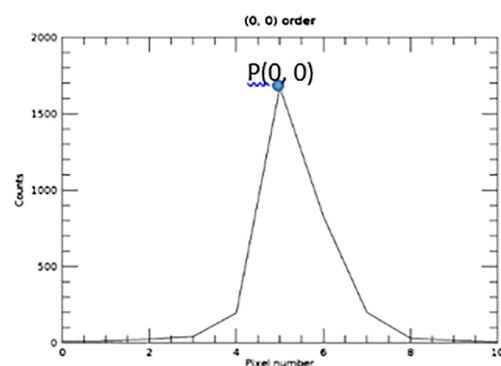

$\mathrm{a}-2$
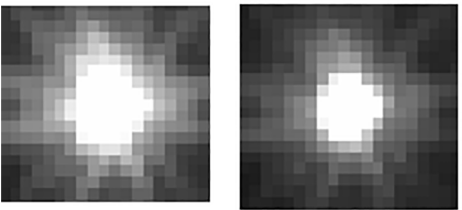

$\mathrm{b}-2$

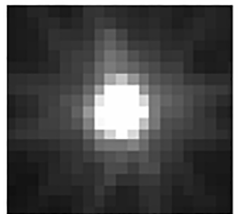

b-3
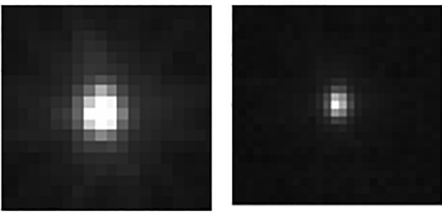

b-4

b-5

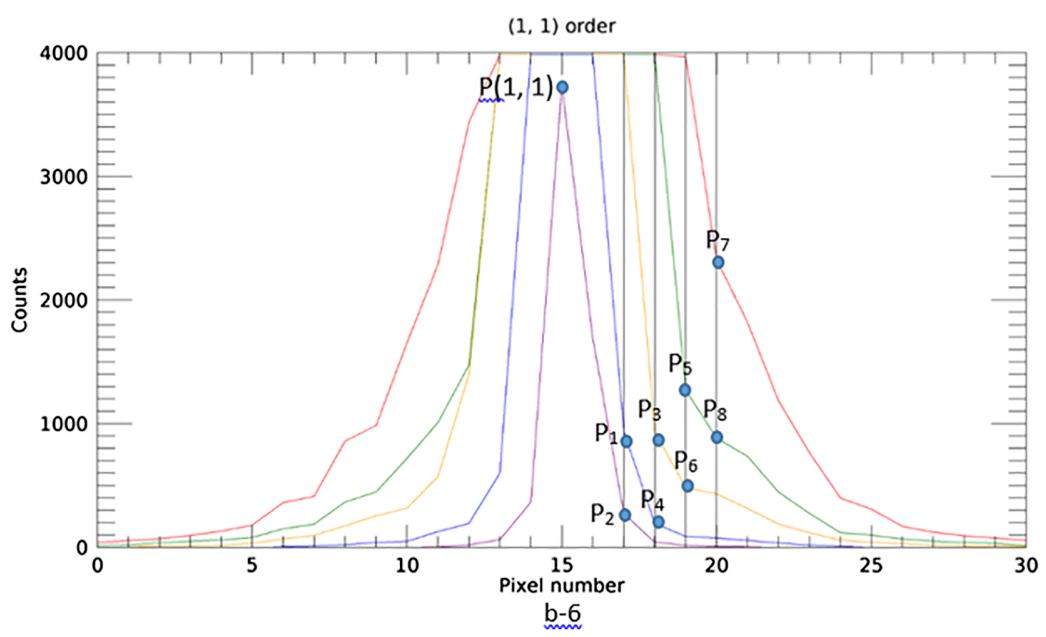

Fig. 14 Ratio of intensities between wanted $(1,1)$ order and unwanted $(0,0)$ order. All plots in this figure are in linear scale. $a-1$ is the PSF of the $(0,0)$ order. $a-2$ is its cross-section profile. $b-1$ is the PSF of $(1,1)$ order taken in the same situation as a-1, meaning the same source intensity, and same exposure time. It is noted that the center is saturated. From b- 1 to b5, the source power and exposure time were gradually reduced $(128 \times)$ until the peak was no longer saturated. b- 6 is the cross section of $b-1$ to $b 5 . P_{1}$ to $P_{8}$ are the points used to calculate the intensity ratio of the $(0,0)$ order and the $(1,1)$ order. 
between the two frames. Finally, the peak intensity of the last frame is multiplied by all the ratios from two consecutive frames, which is the equivalent peak intensity of $(1,1)$ order relative to the peak intensity of the $(0,0)$ order. The final ratio is the peak of the $(0,0)$ order/the equivalent peak of the $(1,1)$ order. Three selected wavelengths have been tested. The results are shown in the following paragraphs.

\subsection{1 $\lambda=1010 \mathrm{~nm}$}

The diffraction efficiency at $\lambda=1010 \mathrm{~nm}$ is one of the lowest in the entire wavelength range. Therefore, the intensity ratio of the wanted $(1,1)$ order to the unwanted $(0,0)$ order is also the lowest. The ratio for this wavelength is important because we want to determine if its intensity is high enough to be used as a guide target. From the standpoint of ghost analysis, it is also the worst offender. Figure 14 shows how the intensity ratio is evaluated.

The data used to evaluate the $(0,0)$ order and $(1,1)$ order intensity ratio are in Table 2.

The equivalent peak intensity of the $(1,1)$ order is $I_{(1,1)}=P_{(1,1)} \cdot \prod_{n}^{i=1} \frac{P_{2 i-1}}{P_{2 i}}$, where $n=4$. The peak intensity of the $(0,0)$ order is $I_{(0,0)}=P_{(0,0)}$. The PSF peak intensity ratio of the wanted $(1,1)$ order to the unwanted $(0,0)$ order is $\frac{I_{(1,1)}}{I_{(0.0)}}=267.08$. The peak count of the $(0,0)$ order is $0.37 \%$ of the peak count of the $(1,1)$ order. The intensity of the $(0,0)$ order is not high enough to be feasible as a guiding target. On the positive side, the low intensity has less impact as a ghost to the wanted spectrum.

Table 2 Data points used for evaluating the intensity ratio.

\begin{tabular}{lcc}
\hline \hline & \multicolumn{1}{c}{$P(1,1)$} & $\frac{3746}{1}$ \\
\cline { 2 - 3 } Peak & $P(0,0)$ & 1673 \\
\hline Pix 17 $(1,1)$ order & P1 & 940 \\
Pix 18 $(1,1)$ order & P2 & 273 \\
& P3 & 935 \\
Pix 19 $(1,1)$ order & P4 & 193 \\
& P5 & 1273 \\
Pix 20 $(1,1)$ order & P6 & 480 \\
& P7 & 2378 \\
\hline \hline
\end{tabular}

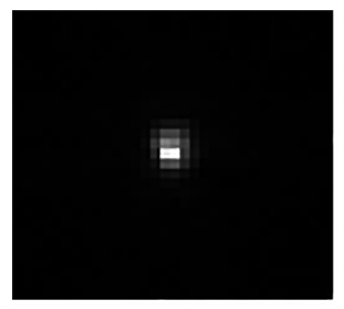

$a-1$

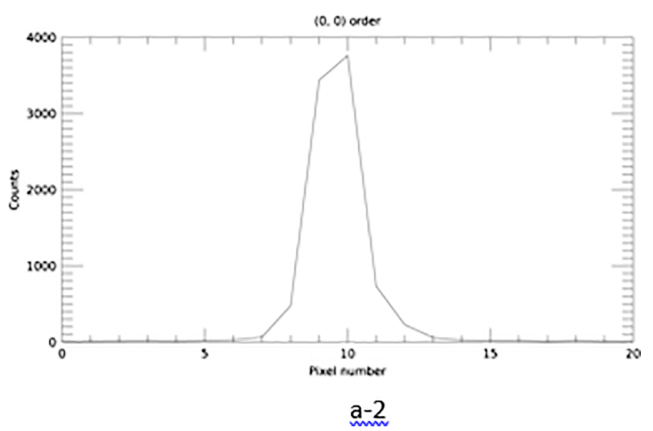

Fig. 15 The PSF of the $(1,1)$ order and its cross-section profile at $1340 \mathrm{~nm}$. It is clear from the PSF that the performance is diffraction limited. 


\subsection{2 $\lambda=1340 \mathrm{~nm}$}

The diffraction efficiency at $\lambda=1340 \mathrm{~nm}$ from both diffractive surfaces is $>95 \%$. The unwanted order $(0,0)$ carries almost 0 power. Even though the exposure time was increased to maximum, the $(0,0)$ order PSF at $1340 \mathrm{~nm}$ was not detected (Fig. 15).

\subsection{3 $\lambda=1580 \mathrm{~nm}$}

The method used for $\lambda=1580 \mathrm{~nm}$ is the same as that used for $1010 \mathrm{~nm}$. Figure 16 shows the images of the wanted $(1,1)$ order and the unwanted $(0,0)$ order.

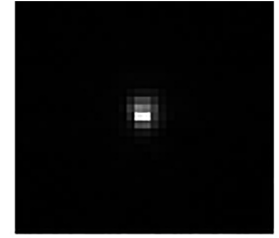

a-1

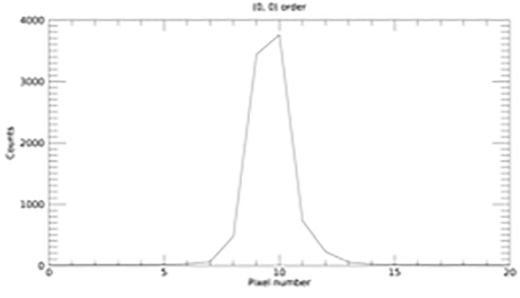

a-2

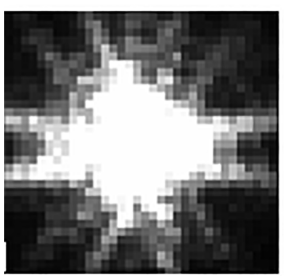

b-1

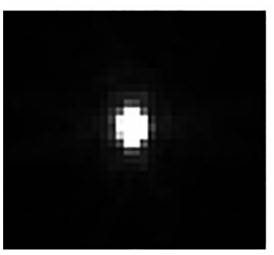

b-5

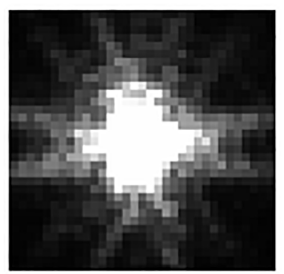

b-2

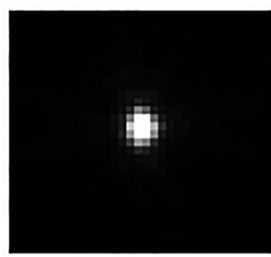

b-6

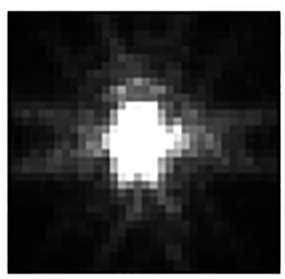

b-3

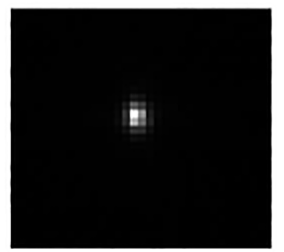

b-7

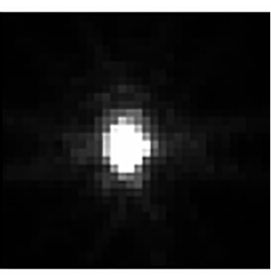

b-4

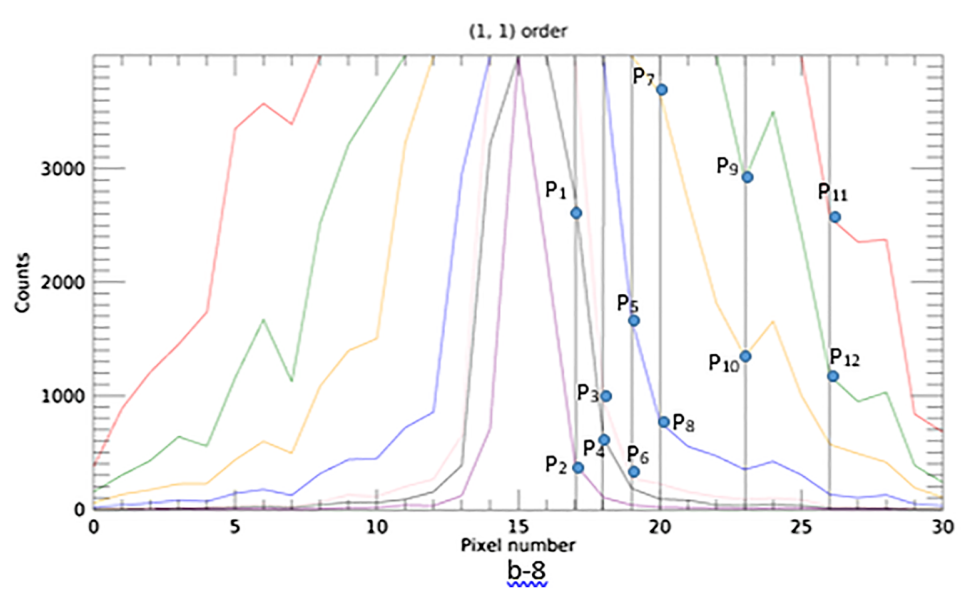

Fig. 16 Ratio of intensities between wanted $(1,1)$ order and unwanted $(0,0)$ order. All plots in this figure are in linear scale. a- 1 is the PSF of the $(0,0)$ order. a- 2 is its cross-section profile. b- 1 is the PSF of $(1,1)$ order taken in the same situation as a-1, meaning the same source intensity, and same exposure time. It is noted that the center is saturated. From b- 1 to b7, the source power and exposure time were gradually reduced until the peak is not saturated. $b-8$ is the cross section of $b-1$ to $b 7$. The $P_{1}$ to $P_{12}$ are the points used to calculate the intensity ratio of $(0,0)$ order and $(1,1)$ order. 
Table 3 Data points used for evaluating the intensity ratio.

\begin{tabular}{lcc}
\hline \hline & $P(1,1)$ & 4095 (slightly saturated) \\
\cline { 2 - 3 } Peak & $P(0,0)$ & 3761 \\
\hline Pix 17 $(1,1)$ order & P1 & 2761 \\
Pix 18 $(1,1)$ order & P3 & 402 \\
Pix 19 $(1,1)$ order & P4 & 964 \\
Pix 20 $(1,1)$ order & P7 & 627 \\
Pix 23 $(1,1)$ order & P9 & 1677 \\
& P10 & 247 \\
Pix 26 (1, 1) order & P11 & 3665 \\
\hline \hline
\end{tabular}

The data used to evaluate the $(0,0)$ order and $(1,1)$ order intensity ratio are in Table 3. The same equation is used to calculate the ratio $I_{(1,1)}=P_{(1,1)} \cdot \prod_{n}^{i=1} \frac{P_{2 i-1}}{P_{2 i}}$, where $n=6$. The PSF peak intensity ratio of the wanted $(1,1)$ order to the unwanted $(0,0)$ order is $\frac{I_{(1,1)}}{I_{(0,0)}}=1594$. That is, the peak counts of the $(0,0)$ order is $0.063 \%$ of the peak counts of the $(1,1)$ order, about $1 / 6$ of ratio found for $1010 \mathrm{~nm}$.

Due to the spectral range of the detector array available at the time of the test, the full spectral range was not able to be tested. The ratio over the entire wavelength range will be measured once we have a detector sensitive over the entire range.

\subsection{Category \#2 PSF from Unwanted Diffraction Orders}

Note: The test in this section is a provisional measurement meant to provide an order of magnitude result for scientists to have a rough estimate on background noise. The next step will be to use the newly delivered, larger IR detector to make a more quantitative measurement, and to use a broadband source to see the effect of the overlapped unwanted orders from different wavelengths.

Category \#2 PSFs are different from category \#1 PSFs in the two aspects: (1) they are created from cross orders. (2) Their spot sizes are much larger than category \#1 PSF's. Usually, the size is a few millimeters even for the shortest wavelength and lowest order combinations. Figure 17 below is a simulated spot diagram with six order combinations using an optical design software Zemax: the wanted $(1,1)$ order and unwanted $(0,0),(1,2),(2,1),(1,0)$, and $(0,1)$ orders for the shortest wavelength at $1010 \mathrm{~nm}$. The $(1,1)$ and $(2,2)$ orders are very small compared to other orders, making them hard to see. The four strongest cross orders, $(1,2),(2,1),(1,0)$, and $(0,1)$ are also shown.

The measured image of the same orders from the EDU is shown in Fig. 18.

Due to project schedule, the EDU grism was not available for detailed testing to quantitatively determine the level of the unwanted orders. Only a qualitative measurement was performed to demonstrate the impact of the unwanted orders. Note: the power carried from $(1,1)$ is more than that from $(0,0)$. This can be seen from Fig. 18 where $(1,0)$ and $(0,1)$ orders have similar intensity as $(1,2)$ and $(2,1)$. However, the desired $(1,1)$ order is much stronger than the $(0,0)$ order. 


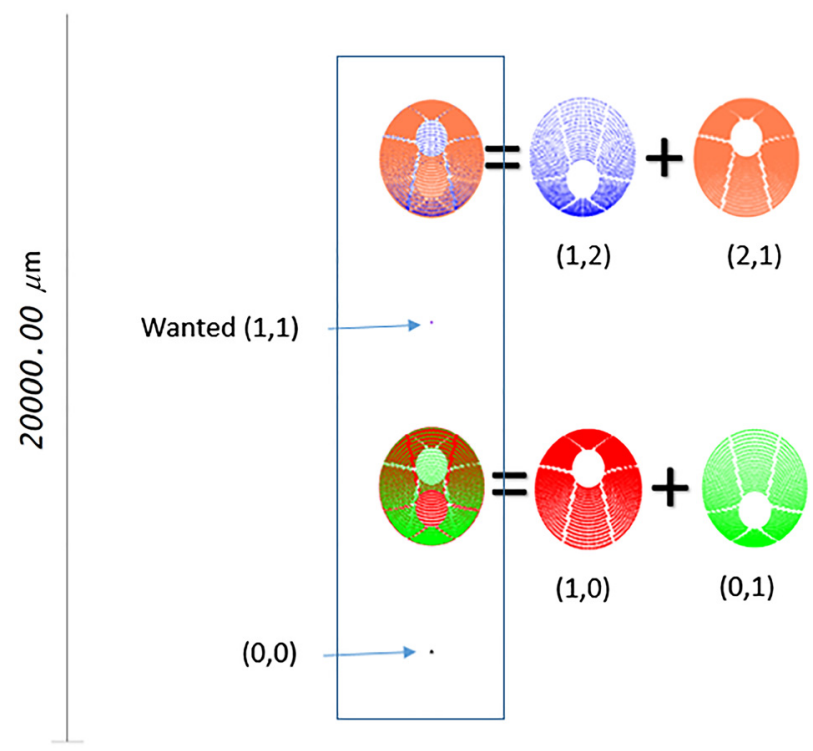

Fig. 17 Spot diagram of the multiple diffraction orders. It is noted that the cross orders $(1,0)$ and $(0,1)$ have the same pattern, but are flipped upside down. The $(1,2)$ and $(2,1)$ orders are similarly flipped. The scale bar on the left is $20-\mathrm{mm}$ long.

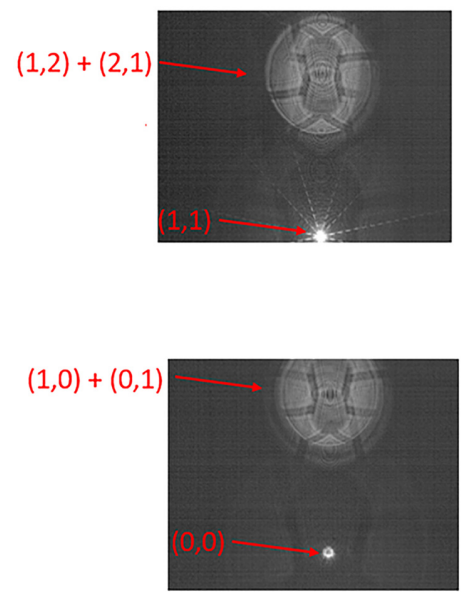

Fig. 18 The real image from the EDU grism. It includes the same number of orders as the simulated spot diagram. The modeled and measured cases are an excellent match.

During the test, the source intensity was first adjusted so that the peak counts of the wanted $(1,1)$ order was $\sim 80 \%$ of detector quantum well. Then the exposure time was increased until the ghost could be seen in a $\log$ scale. The test results are shown in Fig. 19.

The background impact of unwanted orders is limited. Based on these measurements, the intensity of the ghost is $\sim 3$ orders of magnitude lower than the desired $(1,1)$ image. This is the wavelength where the unwanted orders carry the most power.

\section{Summary}

The EDU grism has been tested in ambient conditions with a limited wavelength range of 1000 to $1600 \mathrm{~nm}$ (due to the detector array's spectral response) and at a limited number of field positions (center, $-3 \mathrm{deg}$ in $Y$, and $-10 \mathrm{deg}$ in $X$ ). Performance tests have included diffraction efficiency, dispersion scale, spectral resolving power, and radiometric calibration between the wanted signal and the unwanted ghost orders. All test results suggest that the designed grism 


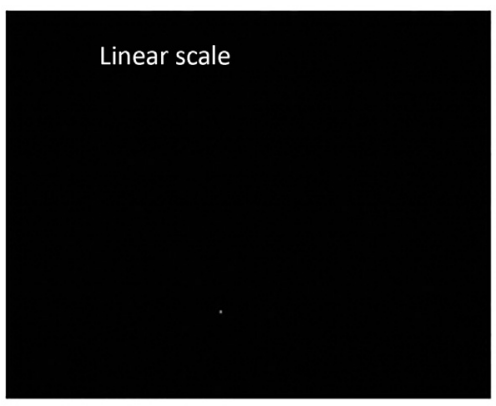

(a)

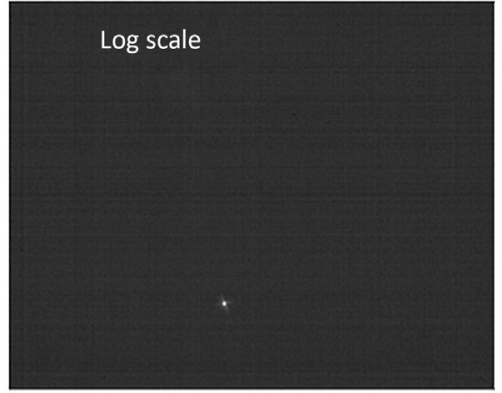

(b)

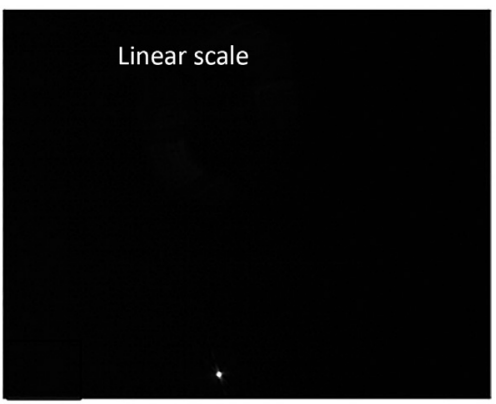

(c)

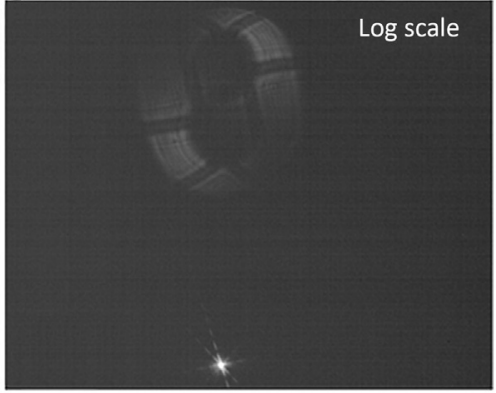

(d)

Fig. 19 (a) and (b) are the unsaturated $(1,1)$ order PSF (80\% quantum well) at $\lambda=1024 \mathrm{~nm}$ in linear and log scales. It is noted that the unwanted $(1,2)$ and $(2,1)$ orders were not detected. (c) and (d) are the same PSF, but the exposure time was increased 16 times. In this situation, the unwanted order was still not visible in the linear scale, but they appear in the log scale display.

specifications have been met for the selected fields and wavelengths in ambient conditions. Even though the encircled energy has not been completed, the small size of the PSF has shown that the diffraction limited performance is achieved.

In the future, additional fields will be measure to cover the entire FOV and the wavelength range will be extended to cover from 1000 to $1930 \mathrm{~nm}$. The goal is to obtain as much information as possible on the ground to make the on-orbit performance well understood. The blue and red edge detection measurement will be added with a precise dispersion scale versus wavelength to achieve the 0.1-nm wavelength accuracy on orbit.

\section{References}

1. "Mission Overview-Roman Space Telescope/NASA," https://roman.gsfc.nasa.gov.

2. Q. Gong et al., "Wide-Field InfraRed Survey Telescope (WFIRST) slitless spectrometer: design, prototype, and results," Proc. SPIE 9904, 990412 (2016).

3. Q. Gong et al., "Wide field slitless spectrometer design for WFIRST," Proc. SPIE to be published (2020).

4. J. Hagopian et al., "Alignment and test of the Wide Field Infrared Survey Telescope (WFIRST) Engineering Design Unit (EDU) grism," Proc. SPIE 11103, 1110306 (2019).

5. C. Schwab et al., "Design of NEID, an extreme precision Doppler Spectrograph for WIYN," Proc. SPIE 9908, 99087H (2016).

6. S. Chamarthi, R. K. Banyal, and S. Sripadmanabhan, "Toward precision radial velocity measurements using Echelle spectrograph at Vainu Bappu Telescope," J. Astron. Telesc. Instrum. Syst. 5(2), 029801 (2019).

7. “SuperK SELECT tunable multi-line filter,’https://www.nktphotonics.com/lasers-fibers/ product/superk-select-multi-line-tunable-filter/.

8. G. J. Swanson, "Binary optical technology: theoretical limits on the diffraction efficiency of multilevel diffractive optical elements," Technical report 914, Lincoln laboratory, MIT (1991). 
Qian Gong received her PhD from the Optical Sciences Center at the University of Arizona in 1990. She is a research optical engineer at NASA Goddard Space Flight Center. She is on the Goddard research engineering partnership program to work with scientists for developing innovative concept design to achieve challenging science goals. Meanwhile, she also designed the Roman grism and prism spectrometers and CGI integral spectrograph. Prior to that, she has worked on HST, JWST, and many other projects as an optical engineer or optics lead.

Matthew Bergkoetter received his BS degree in optical sciences and engineering from the University of Arizona in 2009 and his PhD in optics from the University of Rochester in 2017. He works in wavefront sensing and control at NASA Goddard Space Flight Center.

Joshua Berrier is a graduate with degrees in astronomy/astrophysics, physics, and mathematics. He started his NASA career working as a research associate in the Ultra-Violet and Optical Telescope team investigating supernovae, star clusters, and spiral galaxies for the Swift GammaRay Burst Mission at the Mission Operations Control Center in State College, PA. Since then, he has spent the last decade as an optical engineer working on flight missions at NASA Goddard Flight Space Center.

Victor J. Chambers received his BS degree in optical engineering from the University of Rochester in 2000 and his MS degree in astrophysics from the University of Porto in 2006. He serves as a product delivery lead for the Wide Field Instrument Grism and Prism Assemblies for the Roman Space Telescope mission at the NASA Goddard Space Flight Center, Greenbelt, Maryland, USA.

Margaret Dominguez received her BS degree in physics from the Universidad de las Americas Puebla and her MS and PhD degrees from the College of Optical Sciences at the University of Arizona. She is an optical engineer in the optics branch at NASA Goddard Space Flight Center. She specializes in optical metrology and is currently working on the alignment, integration, and testing of the grism component for the Roman Space Telescope.

Wesley Fincher: Biography is not available.

John Hagopian received his BA degree in astrophysics and his MA degree in nuclear engineering from The University of Virginia. He is an optical physicist and lead engineer at the NASA GSFC delivering instruments for the Cosmic Background Explorer, Broad Band X-Ray Telescope, Hubble, and Cassini Projects. He served as ISIM, OTE, Mission Optical Systems Engineer on the James Webb Space Telescope and on ATLAS. Currently, for the Roman Space Telescope, he is in the role of payload optical systems and alignment engineer.

Catherine Marx received her MS degree in physics from the University of Maryland. She is the optical systems engineer for the Roman Space Telescope Wide Field Instrument and is also an Optics Branch manager at NASA Goddard Space Flight Center. She has extensive experience in optical engineering, especially optical system design and analysis for both space-flight and ground-based optical instrumentation across the electromagnetic spectrum. She completed the optical design of the mid-infrared camera InfraRed Array Camera that flew on the Spitzer Space Telescope.

Laurie Seide received her BS degree in optical engineering and her MS degree in optical sciences from the University of Arizona. She is as an optical analyst for KBR, working at NASA Goddard Space Flight Center. 